\title{
Inequality of Realization of a Stochastic Dynamics Based on the Erdös Discrepancy Problem
}

\author{
Hiroyuki Kato \\ Faculty of Management and Economics, Kaetsu University, Tokyo, Japan \\ Email: hiroyuki-kat0@kaetsu.ac.jp
}

How to cite this paper: Kato, H. (2019) Inequality of Realization of a Stochastic Dynamics Based on the Erdös Discrepancy Problem. Applied Mathematics, 10, 836-847. https://doi.org/10.4236/am.2019.1010060

Received: September 8, 2019

Accepted: October 9, 2019

Published: October 12, 2019

Copyright $\odot 2019$ by author(s) and Scientific Research Publishing Inc. This work is licensed under the Creative Commons Attribution International License (CC BY 4.0).

http://creativecommons.org/licenses/by/4.0/

\begin{abstract}
This paper proposes a stochastic dynamics model in which people who are endowed with different discount factors chose to buy the capital stock periodically with different periodicities and are exposed to randomness at arithmetic progression times. We prove that the realization of a stochastic equilibrium may render to the people quite unequal benefits. Its proof is based on Erdös Discrepancy Problem that an arithmetic progression sum of any sign sequence goes to infinity, which is recently solved by Terence Tao [1]. The result in this paper implies that in some cases, the sources of inequality come from pure luck.
\end{abstract}

\section{Keywords}

Erdös Discrepancy Problem, Arithmetic Progression, Inequality, Economic Dynamics

\section{Introduction}

The existence of inequality of wages, assets, and other incomes in a society has been gaining wide attention recently especially since Pikkety [2] (Atkinson et al. [3], Gabaix et al. [4], Grossman and Helpman [5], Jones [6], Jones and Kim [7], Kasa and Lei [8], Mankiw [9] to name only a few). Many researchers tackled this problem by providing models that explain the empirical data, say, the large gap between capital income and labor one, or inequality among labor incomes, and its extent of that inequality. They employ growth models that endogenously induce the inequality underlining the market mechanism. However, whether the inequality is the problem that needs some remedy or should be taken as mere phenomena depends on the sources of inequality. If the inequality arises from the pure market forces, some people think that interference must be as little as 
possible and the inequality is not a serious problem. If the inequality is born beyond the individual capacity (e.g. inheritance or pure luck), governmental or nongovernmental policies are considered to be required in many respects (tax, wage control, nationalization of institutions and so on) and the inequality is an important problem we must grapple with.

What this paper concerns is the sources of inequality and especially we focus on the possibility that the inequality arises from pure luck. We provide a simple stochastic model in which the ex-post realization of the equilibrium stochastic process is quite biased among people.

To complete this purpose, we have to investigate the existence of some regularity within randomness. Intuitively, the realization of randomness from uniform distribution offers quite equal benefit among people in the long run, for example, in throwing dices or flipping coins, the same numbers realize in almost the same times as experiments continue infinitely. However, from a different mathematical viewpoint, it is possibly said that the same number arises in a regular manner so that the same numbers fall upon almost the same people. To support this aspect, we employ a monumental mathematical theorem which is recently solved. That theorem is the so called Erdös Discrepancy Problem, long time being conjecture from around 1932, which is proved by Terence Tao in [1]. This theorem roughly states that for any random sequence, the realization of which contains almost the same number periodically.

In this paper, we construct a stochastic equilibrium model in which consumers who have different discount factors buy periodically the capital stock so that they are exposed to randomness at arithmetic progression times. Therefore according to the Erdös Discrepancy Problem, there are some people who obtain high wages arbitrary larger times than low wages or who get low wages arbitrary larger times than high wages corresponding to their distinct discount factors. ${ }^{1}$

The main feature in this paper is its approach to elucidating the inequality. The existent models (such as [1]-[9]) basically attribute the inequality to intrinsic character such as productivity, ability and income resource. Since we aim to investigate the other resources that give rise to inequality, the model developed in this paper is in a class of its own though based on standard economics notions such as utility, production and equilibrium, and we draw the distinctive conclusion that the pure randomness possibly causes inequality. The underlying mathematics is the Erdös Discrepancy Problem which is deep and new theorem in the number theory. After Tao's proof [1], some papers clarify the substance of this problem (such as Soundararajan [10]).

The next section describes the stochastic model in which people who have different discount factors select capital stock with different periodicity. The third

${ }^{1}$ The claim that the possession of capital becomes biased among people according to heterogeneous discount factors is apparently related to the Ramsey's conjecture, which says that the people who have the lowest discount factors own all the capital and is solved by many authors in various settings (e.g. Becker [11], Mitra and Sorger [12]). However, in our paper, the discount factor endowed by people who have much capital depends on the realization of stochastic processes and it is not necessarily the lowest discount factor's people who have the large capital. 
section explains the Erdös Discrepancy Problem and applies it to prove the realization of stochastic equilibrium. The last section offers concluding remark.

\section{The Model}

Let $(\Omega, \mathcal{F}, P)$ be a probability space and define a two point valued stochastic process $a_{t} \in\{\bar{a}, \underline{a}\}$ for $t \in \mathbb{N}:=\{1,2, \cdots\}$ with $\bar{a}>\underline{a}>0$. The producers' behavior is described as the following maximization problem.

$$
\max _{L_{t}} a_{t} L_{t}-w_{t} L_{t} .
$$

where $L_{t}$ means the aggregate labor and $w_{t}$ is the wage rate.

We normalize $E_{P}\left[a_{t}\right]=1$.

Consumers buy the capital stock and directly obtain the utility from it and supply labors that yield disutility. Let $x_{t}$ be the quantity of capital and denote the labor supply by $l_{t} \in[0,1]$ at $t$. The quantity of initial capital $x_{1}>0$ decays at the depreciation rate of $0<\delta<1$. So the stock remains like $x_{1}, \delta x_{1}, \delta^{2} x_{1}, \cdots$ as the time passes until the period written by $t=t_{1}$. Consumers buy the new capital and replace the old one at $t=t_{1}+1$. We assume that in the period $t=t_{1}+1$ no capital is available because buying and replacement are assumed to take a time. Next, the new capital is installed after one period at $t_{1}+2$. Then by the same manner $x_{t_{n}+2}, n \in \mathbb{N}$, decays as $x_{t_{n}+2}, \delta x_{t_{n}+2}, \delta^{2} x_{t_{n}+2}, \cdots$ until $t=t_{n+1}$. Consumers buy the new capital and replace the old one at $t=t_{n+1}+1$, and the new capital is installed after one period at $t_{n+1}+2$. So we need $t_{n+1} \geq t_{n}+2$ and the period $\left[t_{n}+2, t_{n+1}\right]$ represents the length of time during which the capital is available. Define for $n \in \mathbb{N}$,

$$
\hat{x}_{t}:=\left\{\begin{array}{ll}
x_{t_{n-1}+2} & t_{n-1}+2 \leq t \leq t_{n} \\
0 & t=t_{n}+1,
\end{array} \quad g(t):= \begin{cases}t-\left(t_{n-1}+2\right) & t_{n-1}+2 \leq t \leq t_{n} \\
0 & t=t_{n}+1\end{cases}\right.
$$

where $t_{0}+2:=1$. Consumers' objective function can be described by

$$
E_{P}\left[\sum_{t=1}^{\infty} \rho^{t-1}\left\{u\left(\delta^{g(t)} \hat{x}_{t}\right)-v\left(l_{t}\right)\right\}\right] .
$$

with $t_{0}+2=1$ where $u$ and $v$ stand for the utility function and disutility one respectively. In what follows, we assume that the utility and disutility functions are linear.

Assumption 1.

$$
u(x)=x, \quad v(l)=\eta l, \quad \eta>0 .
$$

For convenience, we write down the consumers' maximization problem by setting the length of the remaining period of stock, $t_{n}-\left(t_{n-1}+2\right)=: k_{n}$, namely the period between the beginning of newly installed capital, $t_{n-1}+2$ and the end of it, $t_{n}$. Note that the period at which no capital is not yet available is written by $t_{n}+1=\sum_{i=1}^{n}\left(k_{i}+2\right)$. Denote the set of time at which the capital exists by

$$
\mathbb{T}:=\mathbb{N} \backslash\left\{\sum_{i=1}^{n}\left(k_{i}+2\right) \mid n=1,2, \cdots\right\} .
$$


Denote the set of nonnegative integers by $\mathbb{Z}_{+}$(namely $\mathbb{N} \cup\{0\}$ ). Then the consumers' maximization problem is written as follows.

$$
\begin{aligned}
& \max _{\left\{k_{i}\right\}_{i=1,2, \cdots} \subset \mathbb{Z}_{+},\left\{\left(x_{t}, l_{t}\right)\right\}_{t}} \\
& E_{P}\left[x_{1}\left(1+\rho \delta+\rho^{2} \delta^{2}+\cdots+\rho^{k_{1}} \delta^{k_{1}}\right)-\eta\left(l_{1}+\rho l_{2}+\cdots+\rho^{k_{1}} l_{k_{1}+1}\right)\right. \\
& -\rho^{k_{1}+1} \eta l_{k_{1}+2}+\rho^{k_{1}+2} x_{k_{1}+3}\left(1+\rho \delta+\rho^{2} \delta^{2}+\cdots+\rho^{k_{2}} \delta^{k_{2}}\right) \\
& -\rho^{k_{1}+2} \eta\left(l_{k_{1}+3}+\rho l_{k_{1}+4}+\cdots+\rho^{k_{2}} l_{k_{1}+3+k_{2}}\right)-\rho^{k_{1}+2+k_{2}+1} \eta l_{k_{1}+2+k_{2}+2} \\
& +\rho^{k_{1}+2+k_{2}+2} x_{k_{1}+2+k_{2}+3}\left(1+\rho \delta+\rho^{2} \delta^{2}+\cdots+\rho^{k_{3}} \delta^{k_{3}}\right) \\
& -\rho^{k_{1}+2+k_{2}+2} \eta\left(l_{k_{1}+2+k_{2}+3}+\cdots+\rho^{k_{3}} l_{k_{1}+2+k_{2}+3+k_{3}}\right) \\
& -\rho^{k_{1}+2+k_{2}+2+k_{3}+1} \eta l_{k_{1}+2+k_{2}+2+k_{3}+2} \\
& +\cdots+\rho^{\sum_{i=1}^{n}\left(k_{i}+2\right)} x_{\sum_{i=1}^{n}\left(k_{i}+2\right)+1}\left(1+\rho \delta+\rho^{2} \delta^{2}+\cdots+\rho^{k_{n+1}} \delta^{k_{n+1}}\right) \\
& -\rho^{\sum_{i=1}^{n}\left(k_{i}+2\right)} \eta\left(l_{\sum_{i=1}^{n}\left(k_{i}+2\right)+1}+\cdots+\rho^{k_{n+1}} l_{\sum_{i=1}^{n}\left(k_{i}+2\right)+1+k_{n+1}}\right) \\
& \left.-\rho^{\sum_{i=1}^{n}\left(k_{i}+2\right)+k_{n+1}+1} \eta l_{\sum_{i=1}^{n+1}\left(k_{i}+2\right)}+\cdots\right]
\end{aligned}
$$

subject to

$$
\begin{gathered}
S_{t} \Delta \theta_{t}=w_{t} l_{t}, t \in \mathbb{T} \\
x_{t+1}+S_{t} \Delta \theta_{t}=w_{t} l_{t}, t \notin \mathbb{T}
\end{gathered}
$$

where $S_{t}$ is the price of stock, which is used for financing the capital or saving, and $\Delta \theta_{t}$ means the increment of quantity of the stock at $t$. Notice that $x_{t+1}$ is bought at $t$.

We assume that the price of stock has no trend.

\section{Assumption 2.}

$$
E_{P}\left[S_{t}\right]=S_{1}>0
$$

for some $S_{1}>0$.

Thus consumers prefer buying at most capital to saving something at the periods other than $\mathbb{T}$ due to the linearity of utility, presence of discounting $\rho$ and no trend of stock prices. They save only when being in $\mathbb{T}$ and buy the capital using all the savings and current wages while being in other than $\mathbb{T}$. Hence we can express as

$$
x_{k_{1}+3}=S_{k_{1}+2} \sum_{t=1}^{k_{1}+1} \Delta \theta_{t}+w_{k_{1}+2} l_{k_{1}+2}=S_{k_{1}+2} \sum_{t=1}^{k_{1}+1}\left\{\frac{w_{t} l_{t}}{S_{t}}\right\}+w_{k_{1}+2} l_{k_{1}+2},
$$

and

$$
x_{\sum_{i=1}^{n+1}\left(k_{i}+2\right)+1}=S_{\sum_{i=1}^{n+1}\left(k_{i}+2\right)} \sum_{t=\sum_{i=1}^{n}\left(k_{i}+2\right)+1}^{n\left(k_{i}+2\right)+k_{n+1}+1}\left\{\frac{w_{t} l_{t}}{S_{t}}\right\}+w_{\sum_{i=1}^{n+1}\left(k_{i}+2\right)} l_{\sum_{i=1}^{n+1}\left(k_{i}+2\right)}
$$

for $n=1,2, \cdots$. Set the price process $S_{t}$ and $w_{t}$ by for some $\varepsilon>0$,

$$
e S_{t}=w_{t} \text { and } E_{P}\left[w_{t}\right]=1 .
$$

Since it needs to hold $a_{t}=w_{t}$ in equilibrium due to the linearity of produc- 
tion function, putting $E_{P}\left[w_{t}\right]=1$ is required for equilibrium. Next we impose parametric assumptions, which lead to the situation where consumers postpone working as late as possible but cannot help but work when buying capital in the time of the form $\sum_{i=1}^{n}\left(k_{i}+2\right)$.

Let $\bar{\rho}$ be the positive solution to $\bar{\rho}^{2}+(\eta \delta) \bar{\rho}-\eta=0$, namely, $\bar{\rho}=\left(-\eta \delta+\sqrt{(\eta \delta)^{2}+4 \eta}\right) / 2$.

\section{Assumption 3.}

$$
\eta \leq \frac{1}{2(2-\delta)} \text { and } \bar{\rho}>\rho>\frac{\eta}{1+\eta \delta} .
$$

Since $\delta<1$, we have $\eta \leq 1 /(4-2 \delta)<1 /(1+\delta)$. Due to $\eta^{2}+(\eta \delta) \eta-\eta<0$, we have $\bar{\rho}>\eta>\eta /(1+\eta \delta)$. Thus the assumptions are consistent. From the latter part of Assumption 3 and due to $0>\rho^{2}+(\eta \delta) \rho-\eta$, we obtain

$$
\frac{\rho}{1-\rho \delta}>\eta>\frac{\rho^{2}}{1-\rho \delta} \text {. }
$$

Note from (1) and (2) that for $1 \leq t \leq k_{1}+1$, the marginal utility of $l_{t}$ that contributes to $x_{k_{1}+3}$ is $\rho^{k_{1}+2} S_{k_{1}+2} w_{t}\left(1+\rho \delta+\rho^{2} \delta^{2}+\cdots+\rho^{k_{2}} \delta^{k_{2}}\right) / S_{t}$, and marginal disutility is $\rho^{t-1} \eta$.

We calculate as follows; for $1 \leq t \leq k_{1}+1$,

$$
\begin{aligned}
& E_{P}\left[\left(\rho^{k_{1}+2} S_{k_{1}+2} \frac{w_{t}}{S_{t}}\left(1+\rho \delta+\rho^{2} \delta^{2}+\cdots \rho^{k_{2}} \delta^{k_{2}}\right)-\eta \rho^{t-1}\right) l_{t}\right] \\
& \leq E_{P}\left[\left(\rho^{k_{1}+2} S_{k_{1}+2} \frac{w_{t}}{S_{t}}\left(1+\rho \delta+\rho^{2} \delta^{2}+\cdots \rho^{k_{2}} \delta^{k_{2}}\right)-\eta \rho^{k_{1}}\right) l_{t}\right] \\
& =E_{P}\left[\rho^{k_{1}}\left(\rho^{2} S_{k_{1}+2} \varepsilon\left(1+\rho \delta+\rho^{2} \delta^{2}+\cdots \rho^{k_{2}} \delta^{k_{2}}\right)-\eta\right) l_{t}\right] \\
& <E_{P}\left[\rho^{k_{1}}\left(\rho^{2} S_{k_{1}+2} \varepsilon \frac{1}{1-\rho \delta}-\eta\right) l_{t}\right]=\rho^{k_{1}}\left(\rho^{2} \frac{1}{1-\rho \delta}-\eta\right) l_{t}<0 .
\end{aligned}
$$

The second and fourth equalities come from (4) and the last inequality is obtained by (5). Hence consumers select $l_{t}=0$ for $1 \leq t \leq k_{1}+1$. For $\sum_{i=1}^{n}\left(k_{i}+2\right)+1 \leq t \leq \sum_{i=1}^{n}\left(k_{i}+2\right)+k_{n+1}+1, \quad n=1,2,3, \cdots$, the same arguments apply. Hence we conclude that

$$
l_{t}=0 \text { for } t \in \mathbb{T} .
$$

Consider $t=k_{1}+2$. We see from (1) and (2) that the marginal utility of $l_{k_{1}+2}$ that contributes to $x_{k_{1}+3}$ is $\rho^{k_{1}+2} w_{k_{1}+2}\left(1+\rho \delta+\rho^{2} \delta^{2}+\cdots+\rho^{k_{2}} \delta^{k_{2}}\right)$, and marginal disutility is $\rho^{k_{1}+1} \eta$. We calculate as

$$
\begin{aligned}
& E_{P}\left[\left(\rho^{k_{1}+2} w_{k_{1}+2}\left(1+\rho \delta+\rho^{2} \delta^{2}+\cdots+\rho^{k_{2}} \delta^{k_{2}}\right)-\eta \rho^{k_{1}+1}\right) l_{k_{1}+2}\right] \\
& =E_{P}\left[\rho^{k_{1}+1}\left(\rho w_{k_{1}+2}\left(1+\rho \delta+\rho^{2} \delta^{2}+\cdots+\rho^{k_{2}} \delta^{k_{2}}\right)-\eta\right) l_{k_{1}+2}\right] \\
& =\rho^{k_{1}+1}\left(\rho\left(1+\rho \delta+\rho^{2} \delta^{2}+\cdots+\rho^{k_{2}} \delta^{k_{2}}\right)-\eta\right) l_{k_{1}+2} .
\end{aligned}
$$

Therefore if $\rho\left(1+\rho \delta+\cdots+(\rho \delta)^{k_{2}}\right)>\eta$, it follows that $l_{k_{1}+2}=1$, if otherwise, 
$l_{k_{1}+2}=0$ holds. But if $l_{k_{1}+2}=0$, consumers cannot replace the capital so $k_{1}=\infty$, whose case can be neglected because the period $k_{2}$ arising from new capital is not selected at the outset. For $k_{n}+2, n=2,3, \cdots$, the same arguments apply. Thus we see that

$$
l_{t}=1 \text { for } t \notin \mathbb{T} .
$$

Hence from (2) and (3) it holds that for $n=1,2, \cdots$,

$$
x_{\sum_{i=1}^{n}\left(k_{i}+2\right)+1}=w_{\sum_{i=1}^{n}\left(k_{i}+2\right)} .
$$

Thus we have $E_{P}\left[x_{\sum_{i=1}^{n}\left(k_{i}+2\right)+1}\right]=1$ for all $n$. So we can rewrite the objective function in (1) as follows.

Objective function

$$
\begin{aligned}
= & \left(1+\rho \delta+\rho^{2} \delta^{2}+\cdots+\rho^{k_{1}} \delta^{k_{1}}-\rho^{k_{1}+1} \eta\right) \\
& +\rho^{k_{1}+2}\left(1+\rho \delta+\rho^{2} \delta^{2}+\cdots+\rho^{k_{2}} \delta^{k_{2}}-\rho^{k_{2}+1} \eta\right) \\
& +\rho^{k_{1}+2+k_{2}+2}\left(1+\rho \delta+\rho^{2} \delta^{2}+\cdots+\rho^{k_{3}} \delta^{k_{3}}-\rho^{k_{3}+1} \eta\right)+\cdots \\
& +\rho^{\sum_{i=1}^{n}\left(k_{i}+2\right)}\left(1+\rho \delta+\rho^{2} \delta^{2}+\cdots+\rho^{k_{n+1}} \delta^{k_{n+1}}-\rho^{k_{n+1}+1} \eta\right)+\cdots \\
= & \left(1+\rho \delta+\rho^{2} \delta^{2}+\cdots+\rho^{k_{1}} \delta^{k_{1}}-\rho^{k_{1}+1} \eta\right) \\
& +\rho^{k_{1}+2}\left\{\left(1+\rho \delta+\rho^{2} \delta^{2}+\cdots+\rho^{k_{2}} \delta^{k_{2}}-\rho^{k_{2}+1} \eta\right)\right. \\
& +\rho^{k_{2}+2}\left(1+\rho \delta+\rho^{2} \delta^{2}+\cdots+\rho^{k_{3}} \delta^{k_{3}}-\rho^{k_{3}+1} \eta\right)+\cdots \\
& \left.+\rho^{\sum_{i=2}^{n}\left(k_{i}+2\right)}\left(1+\rho \delta+\rho^{2} \delta^{2}+\cdots+\rho^{k_{n+1}} \delta^{k_{n+1}}-\rho^{k_{n+1}+1} \eta\right)+\cdots\right\},
\end{aligned}
$$

Define the value function by

$$
V:=\sup _{\left\{k_{i}\right\}_{i=1,2, \ldots} \subset \mathbb{Z}_{+}} \text {[objective function]. }
$$

We can write

$$
V=\max _{k_{1}}\left[\left(1+\rho \delta+\rho^{2} \delta^{2}+\cdots \rho^{k_{1}} \delta^{k_{1}}-\rho^{k_{1}+1} \eta\right)+\rho^{k_{1}+2} V\right] .
$$

From the recursive character seen above, we see that at the optimal, all $k_{i}, i=1,2, \cdots$ are the same. Write the optimal $k_{i}$ as $k^{*}$. Then the maximal value takes the form

$$
\begin{aligned}
V & =\left(1+\rho \delta+(\rho \delta)^{2}+\cdots+(\rho \delta)^{k^{*}}-\rho^{k^{*}+1} \eta\right)\left[1+\rho^{k^{*}+2}+\rho^{2\left(k^{*}+2\right)}+\rho^{3\left(k^{*}+2\right)}+\cdots\right] \\
& =\frac{1+\rho \delta+(\rho \delta)^{2}+\cdots+(\rho \delta)^{k^{*}}-\rho^{k^{*}+1} \eta}{1-\rho^{k^{*}+2}} .
\end{aligned}
$$

In what follows, we aim to determine the concrete number of $k^{*}$. Let us put

$$
v(k):=\frac{1+\rho \delta+(\rho \delta)^{2}+\cdots+(\rho \delta)^{k}-\rho^{k+1} \eta}{1-\rho^{k+2}} .
$$

Then the optimization problem boils down to 


$$
V=\max _{k} v(k) .
$$

We investigate the difference of the above $v(k)$ with respect to $k$. One has

$$
\begin{aligned}
& v(k+1)-v(k) \\
& =\frac{\left(1-\rho^{k+3}\right)(\rho \delta)^{k+1}+\rho^{k+1} \eta(1-\rho)-\rho^{k+2}(1-\rho)\left(1+\rho \delta+(\rho \delta)^{2}+\cdots+(\rho \delta)^{k+1}\right)}{\left(1-\rho^{k+3}\right)\left(1-\rho^{k+2}\right)} .
\end{aligned}
$$

It suffices to know the sign of the numerator in (9) to determine the sign of the fraction (9). Note that

$$
\begin{aligned}
\operatorname{sign}(v(k+1)-v(k)) \\
=\operatorname{sign}\left\{\left(1-\rho^{k+3}\right)(\rho \delta)^{k+1}+\rho^{k+1} \eta(1-\rho)\right. \\
\left.-\rho^{k+2}(1-\rho)\left(1+\rho \delta+(\rho \delta)^{2}+\cdots+(\rho \delta)^{k+1}\right)\right\} \\
=\operatorname{sign}\left\{\left(1-\rho^{k+3}\right) \delta^{k+1}+\eta(1-\rho)-\rho(1-\rho)\left(1+\rho \delta+(\rho \delta)^{2}+\cdots+(\rho \delta)^{k+1}\right)\right\} .
\end{aligned}
$$

Put

$$
\begin{aligned}
& \Delta(k, k+1) \\
& :=\left(1-\rho^{k+3}\right) \delta^{k+1}+\eta(1-\rho)-\rho(1-\rho)\left(1+\rho \delta+(\rho \delta)^{2}+\cdots+(\rho \delta)^{k+1}\right) .
\end{aligned}
$$

Then we can write as

$$
\operatorname{sign}(v(k+1)-v(k))=\operatorname{sign} \Delta(k, k+1) .
$$

Now we further put the following assumption on the parameters.

\section{Assumption 4.}

$$
\delta<\frac{1-\rho^{3}}{1-\rho^{4}} \text { and } \bar{\rho}>\frac{\eta+\delta}{1-\delta} .
$$

Both inequalities in Assumption 4 are satisfied when $\delta$ is sufficiently small because if $\delta=0$, all inequalities hold consistently with Assumption 3 .

It follows from former part of Assumption 4 that $\delta-1+\rho^{3}(1-\rho \delta)<0$, which leads to $\delta-1+\rho^{k+3}(1-\rho \delta)<0$ for $k=0,1,2, \cdots$, and further we see that

$$
\begin{aligned}
& \delta^{k+1}\left\{\delta-1+\rho^{k+3}(1-\rho \delta)\right\} \\
& =\delta^{k+1}\left\{\left(1-\rho^{k+4}\right) \delta-\left(1-\rho^{k+3}\right)\right\}=\left(1-\rho^{k+4}\right) \delta^{k+2}-\left(1-\rho^{k+3}\right) \delta^{k+1}<0
\end{aligned}
$$

for $k=0,1,2, \cdots$. Thus we find that $\left(1-\rho^{k+3}\right) \delta^{k+1}$ is strictly decreasing function in $k$. So equivalently $\left(1-\rho^{k+3}\right) \delta^{k+1}+\eta(1-\rho)$ is strictly decreasing function in $k$. Together with the fact that $\rho(1-\rho)\left(1+\rho \delta+(\rho \delta)^{2}+\cdots+(\rho \delta)^{k+1}\right)$ is strictly increasing in $k$, we see that

$$
\Delta(k, k+1) \text { is strictly decreasing in } k .
$$

Thus we process the following arguments.

If $v(1)-v(0) \leq 0$, it holds that $v(k+1)-v(k)<0$ for $k=1,2, \cdots$. In this case, we have $k^{*}=0$ since $v$ is decreasing entirely. It requires $\rho>\eta$, which induces $l_{i\left(k^{*}+2\right)}=1, i=1,2, \cdots$. 
If $v(1)-v(0)>0$ and $v(2)-v(1) \leq 0$, it holds that $v(k+1)-v(k)<0$ for $k=2,3, \cdots$. In this case, we have $k^{*}=1$ if $\rho(1+\rho \delta)>\eta$ which leads to $l_{i\left(k^{*}+2\right)}=1, \quad i=1,2, \cdots$.

If $v(2)-v(1)>0$ and $v(3)-v(2) \leq 0$, it holds that $v(1)-v(0)>0$ and $v(k+1)-v(k)<0$ for $k=3,4, \cdots$. In this case, we have $k^{*}=2$ if $\rho\left(1+\rho \delta+(\rho \delta)^{2}\right)>\eta$ which implies $l_{i\left(k^{*}+2\right)}=1, i=1,2, \cdots$.

And so forth $\cdots$. Thus we see that for $k=1,2, \cdots$,

if $v(k)-v(k-1)>0$ and $v(k+1)-v(k) \leq 0$, then we have $k=k^{*}$.

if $\rho\left(1+\rho \delta+(\rho \delta)^{2}+\cdots+(\rho \delta)^{k}\right)>\eta$ which leads to $l_{k^{*}+2}=1, i=1,2, \cdots$.

Note that $\Delta(k, k+1)<0$ for large $k$ because $\left(1-\rho^{k+3}\right) \delta^{k+1}+\eta(1-\rho)$ converges to $\eta(1-\rho)$ and $\rho(1-\rho)\left(1+\rho \delta+(\rho \delta)^{2}+\cdots+(\rho \delta)^{k+1}\right)$ converges to $\rho(1-\rho) /(1-\rho \delta)$ as $k \rightarrow \infty$, and because $\eta(1-\rho)<\rho(1-\rho) /(1-\rho \delta)$ by (5).

Since $\eta \leq 1 /(4-2 \delta)$ in Assumption 3, we have $(\eta \delta)^{2}+4 \eta \leq 1+2 \eta \delta+(\eta \delta)^{2}$, which leads to $-\eta \delta+\sqrt{(\eta \delta)^{2}+4 \eta} \leq 1$. Thus it holds that $\bar{\rho} \leq \frac{1}{2} \quad$ (recall the definition of $\bar{\rho}$ before Assumption 3). We can confirm for $\rho \in(0, \bar{\rho}]$ that

$$
\Delta(k, k+1) \text { is strictly increasing as } \rho \rightarrow 0,
$$

and that for any $k$,

$$
\Delta(k, k+1)>0 \text { holds for small } \rho .
$$

Consider the case of $\bar{\rho}>\rho \geq(\eta+\delta) /(1-\delta)$. Then

$$
\begin{aligned}
\Delta(0,1) & =\left(1-\rho^{3}\right) \delta+\eta(1-\rho)-\rho(1-\rho)(1+\rho \delta) \\
& =(1-\rho)\left(1+\rho+\rho^{2}\right) \delta+\eta(1-\rho)-\rho(1-\rho)(1+\rho \delta) \\
& =(1-\rho)\{\eta+\delta-(1-\delta) \rho\} \leq 0 .
\end{aligned}
$$

Since $\Delta(k, k+1)<0$ for $k=1,2, \cdots$ and $\rho>\eta$, we obtain $k^{*}=0$.

Although $\Delta(1,2)<0$ at $\rho=(\eta+\delta) /(1-\delta)$ (since $k$ rises and (10)), we see from (11) and (12) that $\Delta(1,2)=0$ for some $\rho_{1}<(\eta+\delta) /(1-\delta)$. Since

$$
\begin{aligned}
\Delta(1,2) & =\left(1-\rho_{1}^{4}\right) \delta^{2}+\eta\left(1-\rho_{1}\right)-\rho_{1}\left(1-\rho_{1}\right)\left(1+\rho_{1} \delta+\left(\rho_{1} \delta\right)^{2}\right) \\
& =\left(1-\rho_{1}^{2}\right) \delta^{2}+\eta\left(1-\rho_{1}\right)-\rho_{1}\left(1-\rho_{1}\right)\left(1+\rho_{1} \delta\right)=0,
\end{aligned}
$$

we obtain $\eta-\rho_{1}\left(1+\rho_{1} \delta\right)<0$. Therefore for $(\eta+\delta) /(1-\delta)>\rho \geq \rho_{1}$, we see $\Delta(0,1)>0$ and $\Delta(1,2) \leq 0$ with $\rho(1+\rho \delta)>\eta$. So it follows that $k^{*}=1$ for $(\eta+\delta) /(1-\delta)>\rho \geq \rho_{1}$.

In the same way, we have $\Delta(2,3)=0$ for some $\rho_{2}<\rho_{1}$. Then it holds that $k^{*}=2$ for $\rho_{1}>\rho \geq \rho_{2}$. We have $\Delta(3,4)=0$ for some $\rho_{3}<\rho_{2}$. Then it holds that $k^{*}=3$ for $\rho_{2}>\rho \geq \rho_{3}$, and so on.

If $\rho \leq \eta /(1+\eta \delta)$, which is out of concern due to Assumption 3, it holds $\rho\left(1+\rho \delta+\cdots+(\rho \delta)^{k}\right)<\eta$ for all $k$, which means $k^{*}=\infty$, in other words, this consumer wants to hold the initial stock forever.

To summarize we conclude that $k^{*}=0$ for $\rho \in[(\eta+\delta) /(1-\delta), \bar{\rho}), k^{*}=1$ 
for $\rho \in\left[\rho_{1},(\eta+\delta) /(1-\delta)\right), k^{*}=2$ for $\rho \in\left[\rho_{2}, \rho_{1}\right), \cdots, k^{*}=i$ for $\rho \in\left[\rho_{i}, \rho_{i-1}\right), \ldots$. Since

$$
\begin{aligned}
& \lim _{k \rightarrow \infty} \Delta(k, k+1) \\
& =\lim _{k \rightarrow \infty}\left[\left(1-\rho^{k+3}\right) \delta^{k+1}+\eta(1-\rho)-\rho(1-\rho)\left(1+\rho \delta+(\rho \delta)^{2}+\cdots+(\rho \delta)^{k+1}\right)\right] \\
& =\eta(1-\rho)-\rho(1-\rho) /(1-\rho \delta)<0 \text { for } \rho>\eta /(1+\eta \delta),
\end{aligned}
$$

it follows for some $\underline{\rho}$ that $\rho \downarrow \underline{\rho} \geq \eta /(1+\eta \delta)$ in order for $\Delta(k, k+1)=0$ to hold as $k \rightarrow \infty$ (note $\rho=\eta /(1+\eta \delta)$ is equivalent to $\rho /(1-\rho \delta)=\eta$ ). Hence we have

$$
(\underline{\rho}, \bar{\rho})=\bigcup_{i=0}^{\infty}\left[\rho_{i}, \rho_{i-1}\right)
$$

where $\rho_{0}=(\eta+\delta) /(1-\delta)$ and $\rho_{-1}=\bar{\rho}$.

Let $\varphi_{i+2}:=\left[\rho_{i}, \rho_{i-1}\right)$. A consumer who has a discount factor in $\varphi_{i+2}$ selects $k^{*}=i, i=0,1,2, \cdots$. For people who belong to $\varphi_{i+2}$, the supply of labor is one when $t=(i+2) n, n=1,2, \cdots$, which is the unique opportunity of receiving wages and being exposed by uncertainty, for example, $\varphi_{2}$-people who select $k^{*}=0$ supply one labor at $t=2,4, \cdots, 2 n, \cdots, \varphi_{3}$-people who select $k^{*}=1$ provide one labor at $t=3,6, \cdots, 3 n, \cdots, \varphi_{4}$-people who select $k^{*}=2$ supply one labor at $t=4,8, \cdots, 4 n, \cdots$, and so on. For example, in the case of $t=12$, the prime factorization is $t=2 \times 2 \times 3$ and people who supply one labor are represented by $\{2,3,4,6,12\}$, namely, $6^{\text {th }}$ time of $\varphi_{2}$-people, $4^{\text {th }}$ time of $\varphi_{3}$-people, $3^{\text {rd }}$ time of $\varphi_{4}$-people, $2^{\text {nd }}$ time of $\varphi_{6}$-people and $1^{\text {st }}$ time of $\varphi_{12}$-people are those who supply one labor. Denote the prime factorization of $t$ by

$$
t=\prod_{j=1}^{\omega(t)} p_{j}^{\alpha_{j}}
$$

where $p_{j}$ is a prime number and $\alpha_{j}$ means its multiplicity. Notation $\omega(t)$ obeys the convention in the number theory, which means the number of distinct primes and approximately follows normal distribution (Erdös and Kac Theorem). We write the following expansion as

$$
\begin{aligned}
& \left(1+p_{1}+p_{1}^{2}+\cdots+p_{1}^{\alpha_{1}}\right)\left(1+p_{2}+p_{2}^{2}+\cdots+p_{2}^{\alpha_{2}}\right) \cdots\left(1+p_{\omega(t)}+p_{\omega(t)}^{2}+\cdots+p_{\omega(t)}^{\alpha_{\omega(t)}}\right) \\
& =: 1+x_{1}+x_{2}+\cdots+x_{M(t)}
\end{aligned}
$$

where $M(t):=\left(1+\alpha_{1}\right)\left(1+\alpha_{2}\right) \cdots(1+\omega(t))-1$. Denote the set of label of people who supply one labor at $t$ by

$$
J(t):=\left\{x_{1}, x_{2}, \cdots, x_{M(t)}\right\}
$$

In the case of aforementioned example $t=12$, one see that $\left(1+2+2^{2}\right)(1+3)=1+2+3+2^{2}+6+2^{2} \times 3$. So we get $J(t)=\{2,3,4,6,12\}$ that stands for the set who supply one labor as before. Therefore we have

$$
L_{t}=\# J(t)=M(t) .
$$

Since labor demand is arbitrary from linearity, the supply of labor $\# J(t)=M(t)$ is always in equilibrium of the labor market. The goods equilibrium condition is 
denoted by

$$
\text { supply } a_{t} M(t)=\text { demand } x_{t+1} M(t) \text {. }
$$

Because $a_{t}=w_{t}=x_{t+1}$ (note $x_{t+1}$ is available from $t+1$ but bought at $t$ ), its condition holds. The stock market equilibrium condition is written as

$$
\begin{aligned}
& \text { supply } \frac{w_{t}-x_{t+1}}{S_{t}} M(t) \\
& =\text { demand } \frac{w_{t}}{S_{t}} \cdot[\text { the total number of labor other than } J(t)] .
\end{aligned}
$$

Because $w_{t}=x_{t+1}$ and the labor other than $J(t)$ equals 0 (for each consumer, labor supply is zero in $\mathbb{T}$ ), the above condition follows.

\section{Realization of Stochastic Capital Process}

This section concerns the realization of stochastic capital process. The aggregate capital process in equilibrium is described by

$$
a_{t} M(t)
$$

as in the previous section where $a_{t}\left(=w_{t}\right)$ is the exogenous productivity stochastic process taking value $\bar{a}$ or $\underline{a}$, and $M(t)$ stands for a deterministic one endogenously determined in equilibrium. However, each individual consumer potentially face and really encounter at arithmetic progression times the exogenous stochastic productivity process (equivalently wages) $\left\{a_{1}, a_{2}, a_{3}, \cdots\right\}$, which is realized as $(\bar{a}, \underline{a}, \underline{a}, \bar{a}, \underline{a}, \cdots)$, or $(\bar{a}, \bar{a}, \underline{a}, \bar{a}, \underline{a}, \cdots)$, or $(\underline{a}, \underline{a}, \bar{a}, \underline{a}, \bar{a}, \cdots)$, and so on.

At this point, we introduce the monumental mathematical theorem, known as Erdös Discrepancy Problem, long time being conjecture from around 1932, proved by Terence Tao in (2016). It states that for any sign sequence $f: \mathbb{N} \rightarrow\{-1,1\}$,

$$
\sup _{n, d \in \mathbb{N}}\left|\sum_{j=1}^{n} f(j d)\right|
$$

is infinite. Formally, for any $C>0$ and $f$, there exist $n$ and $d$ such that

$$
\left|\sum_{j=1}^{n} f(j d)\right| \geq C .
$$

Roughly speaking, given infinite sign sequence, say, $\{-1,-1,+1,-1,-1,+1, \cdots\}$, pick up each number skipping $d-1$ times (e.g. pick up $+1,+1, \cdots$ skipping 2 times (avoiding $-1,-1)$ ), which adds up to sufficiently large for sufficiently large length of numbers. This topic generally concerns the problem as to whether there exists some regularity within random sequences. Van der Waerden's theorem (1927) asserts that for any $f$ and $k \in \mathbb{N}$, there exist $a$ and $r \in \mathbb{N}$ such that

$$
f(a)=f(a+r)=f(a+2 r)=\cdots=f(a+(k-1) r)
$$

namely, for any sign sequence there exists any long arithmetic progression with the same number. Erdös Discrepancy Problem says the similar statements that taking a homogeneous arithmetic progression, the either sign outnumbers the other one by arbitrary large extent. 
We apply this Erdös Discrepancy Problem to the exogenous $\left\{a_{t}\right\}$, which is equal to $\left\{w_{t}\right\}$ in equilibrium. In the model in the previous section, consumers are exposed to randomness periodically, namely, $\varphi_{i+2}$-people encounter the randomness at $(i+2) n, n \in \mathbb{N}$ periods for $i \in \mathbb{Z}_{+}$. By redefining $f: \mathbb{N} \rightarrow\{\bar{a}, \underline{a}\}$ and reinterpret $(i+2)=d$, we can say the following theorem.

Theorem. For arbitrary large number, $C>0$, and any realization of $\left\{a_{t}\right\}$, there exists a long period of time, $N \in \mathbb{N}$, and $\varphi_{i+2}$-people who face the high wages or low wages for periods that outnumber the other ones by difference $C>0$, namely,

$$
\begin{aligned}
& \varphi_{i+2} \text {-people encounter } \\
& \left\{\begin{array}{l}
\# \bar{a}-\# \underline{a} \geq C(\text { lucky people case }) \\
\text { or } \\
\# \underline{a}-\# \bar{a} \geq C(\text { unlucky people case })
\end{array}\right.
\end{aligned}
$$

for $t=1,2, \cdots, N$

Roughly speaking, even under random environment, there may be a fixed member in a society who is almost always lucky or unlucky for large period of time. Note that in the case of $d=0$ that attains the given $C$, we take periods, say, $t=2,4,6, \cdots, 2 n, \cdots$ that $\varphi_{2}$-people encounter and reinterpret it as original sequence, then we can take subsequence that attains the given $C$.

\section{Conclusions}

This paper proposes a stochastic dynamics in which people who are endowed with different discount factors buy the capital stock periodically and are exposed to randomness at arithmetic progression times. We prove that the realization of the stochastic equilibrium may render to the people quite unequal benefits. Its proof is based on Erdös Discrepancy Problem that an arithmetic progression sum of any sign sequence goes to infinity, which is recently solved by Terence Tao (2016). There are some people who obtain high wages arbitrary larger times than low wages or who get low wages arbitrary larger times than high wages corresponding to their distinct discount factors. The result in this paper implies that in a certain society, the sources of inequality come from pure luck.

Finally we note the topics that remain in future research. Inequality arising from realization of stochastic processes only identifies the most lucky or the least one and does not explain the distribution of various income realization. In addition, whether people face the fortunate case or not reflects observation of the finite time and we cannot say anything about what occurs beyond the periods. The type of phenomena that is in this paper out of scope may be explained by other approach or more generalized mathematical theorem on the number theory or stochastic analysis.

\section{Conflicts of Interest}

The author declares no conflicts of interest regarding the publication of this paper. 


\section{References}

[1] Piketty, T. (2014) Capital in The Twenty-First Century. Translated by Arthur Goldhammer, The Belknap Press of Harvard University Press, Cambridge, MA.

[2] Tao, T. (2016) The Erdös Discrepancy Problem. Discrete Analysis, 1-27. https://doi.org/10.19086/da.609

[3] Atkinson, A.B., Piketty, T. and Saez, E. (2011) Top Incomes in the Long Run of History. Journal of Economic Literature, 49, 3-71. https://doi.org/10.1257/jel.49.1.3

[4] Gabaix, X., Lasry, J.M., Lions, P.L., Moll, B. and Qu, Z. (2016) The Dynamics of Inequality. Econometrica, 84, 2071-2111. https://doi.org/10.3982/ECTA13569

[5] Grosman, G.M. and Helpman, E. (2018) Growth, Trade, and Inequality. Econometrica, 86, 37-83. https://doi.org/10.3982/ECTA14518

[6] Jones, C.I. (2015) Pareto and Piketty: The Macroeconomics of Top Income and Wealth Inequality. Journal of Economic Perspectives, 29, 29-46.

https://doi.org/10.1257/jep.29.1.29

[7] Jones, C.I. and Kim, J. (2018) A Schumpeterian Model of Top Income Inequality. Journal of Political Economy, 126, 1785-1826. https://doi.org/10.1086/699190

[8] Kasa, K. and Lei, X. (2018) Risk, Uncertainty and the Dynamics of Inequality. Journal of Monetary Economics, 94, 60-78.

https://doi.org/10.1016/j.jmoneco.2017.11.008

[9] Mankiw, N.G. (2015) Yes, $r>g$. So What? American Economic Review, 105, 43-47. https://doi.org/10.1257/aer.p20151059

[10] Soundararajan, K. (2018) Tao's Resolution of the Erdös Discrepancy Problem. Bulletin of the American Mathematical Society, 55, 81-92. https://doi.org/10.1090/bull/1598

[11] Becker, R.A. (1980) On the Long-Run Steady State in a Simple Dynamic Model of Equilibrium with Heterogeneous Households. The Quarterly Journal of Economics, 95, 375-382. https://doi.org/10.2307/1885506

[12] Mitra, T. and Sorger, G. (2013) On Ramsey's Conjecture. Journal of Economic Theory, 148, 1953-1976. https://doi.org/10.1016/j.jet.2013.05.003 